\title{
Camel Milk and the Prevention of Glucose Cataract, an Organ Culture Study
}

\author{
Ali H.S. Alghamdi ${ }^{1}$, Hasabelrasoul Mohamed ${ }^{2}$, Jonathan Austin ${ }^{3, \dagger}$, Collin Henry ${ }^{3, \dagger}$, \\ Kayla Massey ${ }^{3, \dagger}$, Shanzeh Sayied ${ }^{3, \dagger}$, Samiyyah Sledge ${ }^{3, \dagger}$, Aliza Williams ${ }^{3, \dagger}$ and \\ Douglas Borchman ${ }^{3, *}$
}

\author{
${ }^{1}$ Department of Ophthalmology, Faculty of Medicine, Al Baha University, Saudi Arabia \\ ${ }^{2}$ Department of Basic Medical Sciences, Faculty of Applied Medical Sciences, Al Baha University, Saudi \\ Arabia \\ ${ }^{3}$ Department of Ophthalmology and Visual Sciences, University of Louisville, United States of America
}

\begin{abstract}
Purpose: To test if camel milk affects glucose-induced opacity in organ cultured rat and human lenses.
Methods: Whole human and rat lenses were cultured in various media containing either $55 \mathrm{mM}$ glucose, camel milk, or a combination of both glucose and milk. Some lenses were cultured in a media containing neither moiety to establish a control. Absorbance spectra of human and rat lenses were measured daily using a visible/ultraviolet light spectrometer. Lens opacities were graded by a blinded grader from photographs taken daily. Aldose reductase activity, catalase activity, glutathione and receptor for advanced glycation end products levels were assayed.

Results: The optical density and light scattering intensity of human lenses cultured with glucose were higher after two to four days in organ culture compared with lenses cultured without glucose. Camel milk in the culture media attenuated the glucose-induced increase in optical density, light scattering intensity and opacity grade after two to four days for both human and rat lenses. Aldose reductase activity, catalase activity and glutathione levels were restored but the receptor for advanced glycation end products was similar in rat lenses cultured with glucose compared with those cultured with glucose and camel milk. There were no differences between the assayed moieties in human lenses cultured with glucose or glucose plus milk. Since camel milk restored rat lens glutathione levels, it is possible that camel milk may protect the lens from oxidation and significantly reduce the glucose-induced increase in light scattering of human lenses. Structurally and physiologically, rat lenses are distinct from human lenses, therefore, the rat lens data was highly variable when compared with the human lens data, highlighting the importance of using human lenses in future studies.

Conclusions: Camel milk present in the organ culture medium inhibited the glucose-induced opacity in human lenses and restored the amount of glutathione to the same levels of lenses not cultured in glucose. The positive results of the current study leads to future studies to determine the moieties in camel milk that are responsible for cataract inhibition and in vivo studies involving camel milk.
\end{abstract}

Keywords: Camel milk, Cataract, Glucose, Human, Lens, Organ culture.

\section{INTRODUCTION}

Cataract is among the major causes of visual impairment and blindness worldwide [1]. A major goal then is to delay the onset of cataracts. Most cataracts appear in elderly individuals, but diabetes is also a major factor; individuals with diabetes are two times more likely to have cataracts than those without diabetes [2]. In this study, we tested if camel milk inhibited cataract in human and rat lenses in vitro. We chose to study camel milk since it has proven to be beneficial to human health and contains the antioxidant ascorbate at three times the levels found in cow's milk [3-6]. Epidemiological literature suggests that the risk of cataract can be diminished by diets that are optimized for vitamin C, lutein/zeaxanthin, B vitamins, omega-3

\footnotetext{
*Address correspondence to this author at the Kentucky Lions Eye Center, 301 E. Muhamad Ali Boulevard, Louisville, KY 40202, USA; Tel: 011-502-852-7435; Fax: 011-502-852-6584; E-mail: Borchman@louisville.edu

${ }^{\dagger}$ Contributed equally, in alphabetical order.
}

fatty acids, multivitamins, carbohydrates and micronutrients [7]. The health benefits of drinking camel milk, especially for children, has been reviewed [8]. Camel milk consumption lowers the dose required to maintain glycemic control in patients with diabetes [9] and could be an alternative for cow milk allergy in children [10]. Camel milk could also be antimicrobial since the level of lactoferrin, an antimicrobial protein, is higher in camel milk when compared with cows' milk [11]. Camel milk fat globular membrane proteins have been characterized $[12,13]$ and have multiple health benefits for humans $[14,15]$. For example, lactadherin found in milk prevents symptomatic rotavirus infection in breastfed infants [16].

A method to measure absorbance and light scattering for the first time in organ cultured lenses was developed. It was found that light scattering accounted for all of the absorbance of the human lens above 550 $\mathrm{nm}$ which indicates that cellular and molecular structure contributes to the attenuation of light passing through 
the lens rather than the absorbance of unique moieties [17]. Using the new method, we found dexamethasone treated lenses scattered more light when compared with untreated human and rat lenses [17]. Relevant to the present study, almost all of the attenuation of light through glucose treated human lenses was due to light scattering from structural changes [18]. Some of the structural changes in rat lenses cultured with glucose $(\mathrm{RLg})$ may have been caused by oxidative stress since the levels of glutathione, catalase, and aldose reductase were reduced in $\mathrm{RLg}$ compared with rat lenses that were cultured in control media (RLC) [18].

Spectroscopic measurement offers two major advantages over grading of lenses from photographs. Photographic grading only quantifies opacity into 4 to 5 grades, whereas spectroscopic measurement provides a continuous grade and is not subjective. Additionally, one can discern from the absorbance measurements of chromophores whether compositional changes occur; light scattering can also measure changes in lens structure whereas, photographic grading cannot. Another general advantage of spectroscopy is that the sample is not altered or destroyed by spectroscopic measurement so subsequent metabolomic assays can be performed. Finally, another advantage of spectroscopic measurement is unlike metabolomics assays, it can be made continuously on the same lens which is helpful in determining the optimum time point to measure metabolic changes using destructive assays.

Thus, to utilize the advantages listed above, in this study, the opacity of whole human and rat lenses cultured with and without glucose, with and without camel milk and camel milk plus glucose were measured to test the ability of camel milk to ameliorate glucose-induced lens opacity.

\section{METHODS}

The methods used in this study were identical to those described previously $[17,18]$ except contralateral human lenses were incubated with $55 \mathrm{mM}$ glucose, camel milk and glucose plus camel milk.

Unpasteurized camel milk was obtained from Desert Farms, Santa Monica, California, USA. The milk was shipped on ice and stored at $2.8^{\circ} \mathrm{C}$ and used within three weeks. One lot was used for all of the studies. The supplier indicated that $240 \mathrm{~mL}$ of milk contained: $4.5 \mathrm{~g}$ total fat ( $3 \mathrm{~g}$ saturated), $15 \mathrm{mg}$ cholesterol, $150 \mathrm{mg}$ sodium, $390 \mathrm{mg}$ potassium, $11 \mathrm{~g}$ of carbohydrate, $5 \mathrm{~g}$ protein and the following percent of the required amount of vitamins and minerals based on a 2,000 calorie diet: vitamin A $4 \%$, vitamin C $0 \%$, vitamin B1 $70 \%$, calcium $30 \%$, iron $2 \%$, vitamin D $6 \%$, thiamin 70 $\%$, niacin $8 \%$, phosphorous $15 \%$, magnesium $4 \%$ and zinc $4 \%$.

\section{Lens Dissection}

Clear human lenses were obtained from the Kentucky Lions Eye Bank (Louisville, Kentucky, USA) which had been previously dissected from deceased donors. Donors with diabetes or opaque lenses were excluded. All human lenses were collected with written informed consent. This study was approved by the University of Louisville Institutional Review Board. All experiments were performed in accordance with the Declaration of Helsinki. Human lenses were removed from the globe by an anterior approach after removal of the cornea for transplantation. The iris and ciliary body were carefully removed from the lens by cutting the zonules with curved microscissors.

Male Sprague Dawley rats which were approximately eight weeks old and weighing 250 to 274g-each, were euthanized with $\mathrm{CO}_{2}$ and their globes were immediately removed. The animal protocols were approved by the University of Louisville Institutional Animal Care and Use Committee and conformed to the ARVO Statement for the Use of Animals in Ophthalmology and Vision Research. Lenses were dissected using a posterior approach. A small, shallow incision was made at the optic nerve with a scalpel blade and the sclera was cut into four sections with straight micro scissors and peeled back to the iris. The dissection was aided by an illuminated head magnifier (SE MH1047L, Amazon Inc, Seattle Washington, USA). The iris and ciliary body were carefully removed from the lens by cutting the zonules with curved microscissors.

\section{Whole Lens Organ Culture}

Immediately after dissection, human and rat lenses were carefully placed into $10 \mathrm{~mL}$ and $2 \mathrm{~mL}$ of medium, respectively, using curved blunt forceps. The incubation medium was freshly prepared by mixing minimum essential medium (MEM) Eagle with Earle's BSS without L-glutamine or phenol red (Lonza, Walkerville, MD, USA) with L-Glutamine-Penicillin, streptomycin and amphotericin B (Sigma Chemical Company, St Louis, MO, USA) at concentrations of 2 $\mathrm{mM} / \mathrm{mL}, \quad 100 \mathrm{U} / \mathrm{mL}, \quad 0.1 \mathrm{~g} / \mathrm{mL}$, and $0.25 \mu \mathrm{g} / \mathrm{mL}$, 
respectively. The medium used for glucose treated lenses also contained $55 \mathrm{mM}$ glucose. The medium containing camel milk had the same ingredient concentrations as stated above but with $50 \%$ fresh camel milk (Desert Farms, Santa Monica, California, USA). The lenses were incubated at $37^{\circ} \mathrm{C}$ in a $5 \% \mathrm{CO}_{2}$ atmosphere. The lens culture medium was replaced every day. Lenses were incubated for a period of four days. An incubation period of four days was chosen because after this period of time, control lenses became increasingly opaque $[17,18]$ and it was one of the earliest times in which there was a significant difference in the optical properties of lenses cultured with glucose compared to those cultured without glucose [18].

\section{Lens Optical Property Measurements}

Immediately after dissection, lenses were photographed and lens absorbance and light scattering were measured every 24 hours as described [18].

\section{Human Lens Assays}

Human lenses were homogenized in $0.5 \mathrm{ml}$ of phosphate buffered saline (Sigma Chemical Company, St Louis, MO, USA) and centrifuged to obtain a clear supernatant. Aliquots of the homogenate $(55 \mu \mathrm{L})$ were used to measure: glutathione (Kit CS0260, SigmaAldrich Inc., St Louis MO, USA), catalase (Kit CAT100, Sigma-Aldrich Inc., St Louis MO, USA), aldose reductase (United States Biological, Salem MA, USA) and a receptor for advanced glycation end products (RAGE) (ELISA Kit RAB1040, Sigma-Aldrich Inc., St Louis MO, USA) according to the manufacturer's instructions.

Averages were compared using the Student's t-test. Data were reported as averages \pm the standard error of the mean.

\section{RESULTS}

\section{Human Lens Studies}

The demographics of ten human lenses obtained from five donors are listed in Table 1. A broad absorbance peak near $600 \mathrm{~nm}$ appeared in the glucose cultured human $(\mathrm{HLg})$ spectra that was not present in the human control (HLc) and glucose plus camel milk cultured human (HLgm) spectra (Figure 1A). The average level of light scattering was significantly more than two times higher $(P<0.001)$ in HLg compared with HLc (Figure 2C). The optical density (O.D., Figure 2B), and light scattering (Figure 2C) of HLgm was not significantly different $(P>0.05)$ from that of HLC.

When the baseline absorbance at $750 \mathrm{~nm}$ was subtracted from the O.D. at $450 \mathrm{~nm}(\Delta$ O.D.) the $\Delta$ O.D. was higher in $\mathrm{HLg}$ above day two in organ culture compared with HLc, and the $\triangle$ O.D. for HLgm were below that of HLc (Figure 2A).

Human lens opacity was graded into four categories by a blinded grader based on the opacity of the lens (Figure 3A). HLg were almost completely opaque after three days in organ culture (Figure 2D) and more opaque than HLc above zero days in organ culture. After one day, the grade for HLgm reached a maximum that was below the maximum opacity of HLg (Figure 2D).

There was little difference between aldose reductase activity (Figure 4A), the level of the receptor for advanced glycation end products (RAGE) (Figure 4B), Catalase activity (Figure 4C) or glutathione levels (Figure 4D) between HLg and HLgm.

\section{Rat Lens Studies}

The O.D. of rat lenses was greater in wavelengths below $750 \mathrm{~nm}$ in the average spectrum of $\mathrm{RLg}$

Table 1: Demographics and Parameters Related to Human Lens Donors

\begin{tabular}{|c|c|c|}
\hline Donor Parameter & $\begin{array}{c}\text { Control Average } \pm \text { Standard } \\
\text { Deviation }\end{array}$ & $\begin{array}{c}\text { Glucose/Camel Milk Cultured Average } \pm \\
\text { Standard Deviation }\end{array}$ \\
\hline \hline Average Age (years) & $59 \pm 7$ & $39 \pm 9$ \\
\hline Age range & 48 to 65 & 60 \\
\hline Male (\%) & 80 & Caucasian 100 \\
\hline Race (\%) & Caucasian 80, Black 20 & $7 \pm 3$ \\
\hline Average Time from death to enucleation (hours) & $11 \pm 4$ & $0.22 \pm 0.02$ \\
\hline Average lens weight (g) & $0.23 \pm 0.04$ & 10 \\
\hline Number of lenses & 16 & \\
\hline
\end{tabular}



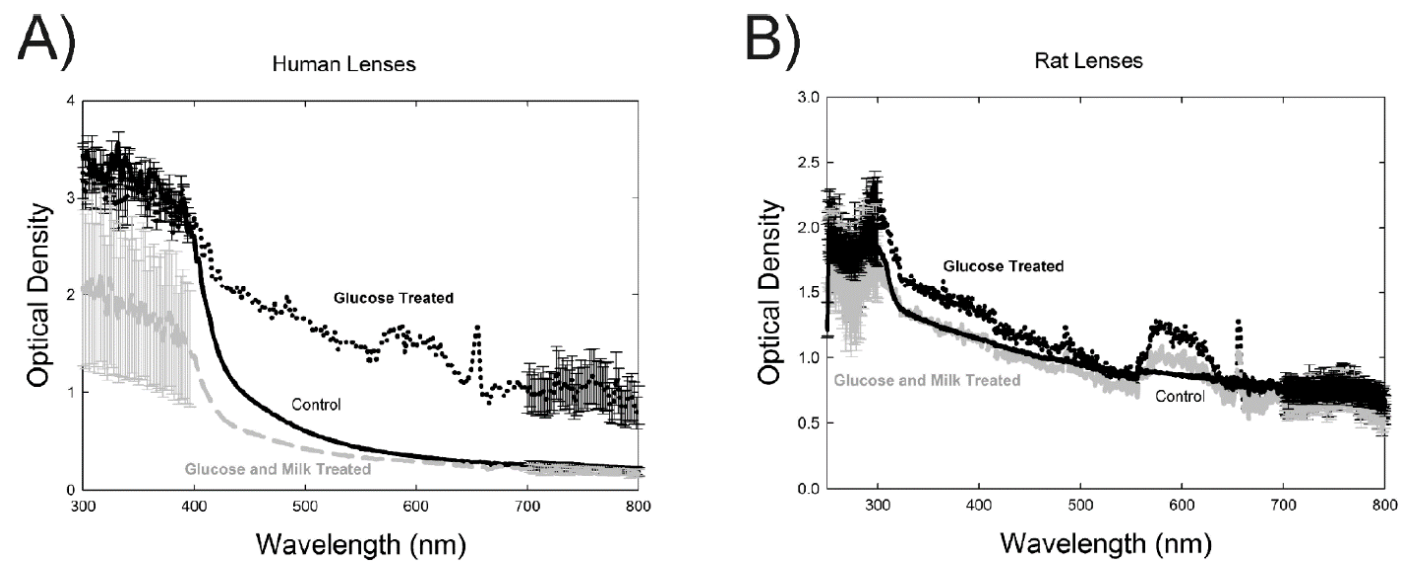

Figure 1: Average absorbance spectra of $\mathbf{A}$ ) human lenses and $\mathbf{B}$ ) rat lenses after three days in organ culture media. ( - ) Untreated lenses. (---) Treated with glucose and milk. (dotted) Treated with glucose. Error bars are the standard error of the mean and are shown only from 700 to $800 \mathrm{~nm}$ and 250 to $300 \mathrm{~nm}$ so as not to obscure the spectra. The error was greatest at lower wavelengths were very little of the light was transmitted. The spectra of human untreated, glucose treated and glucose and milk treated lenses were averaged from 10,5 and 5 spectra, respectively. The spectra of rat untreated, glucose treated and glucose and milk treated lenses were averaged from 19, 8 and 9 spectra, respectively.

A)

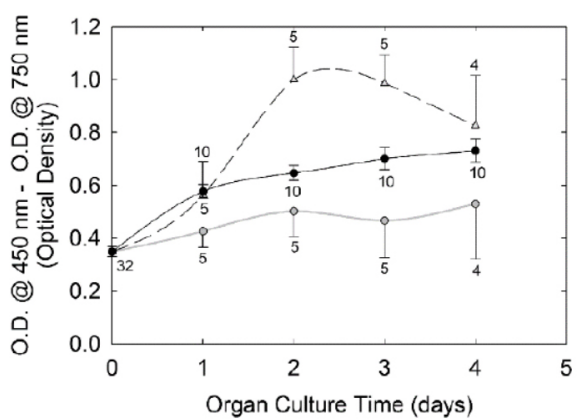

\section{C)}

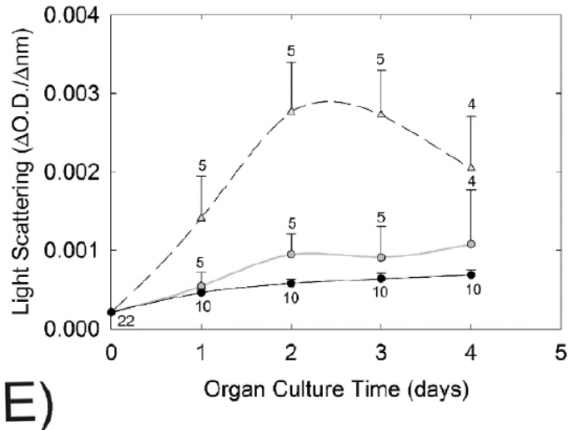

E)

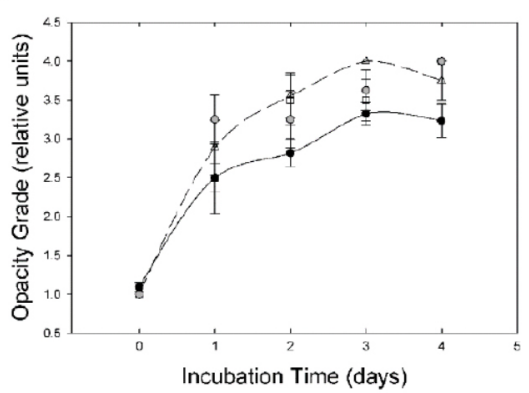

B)

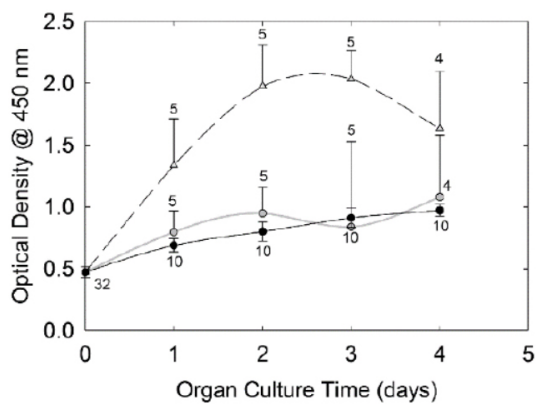

D)

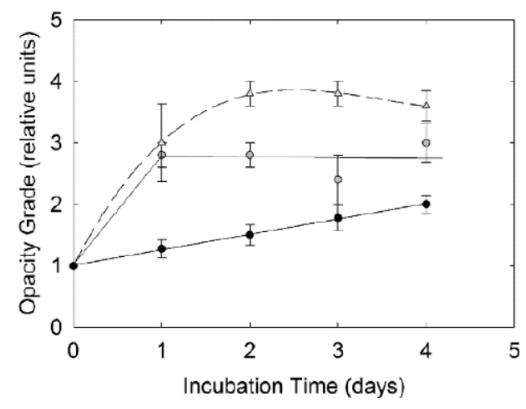

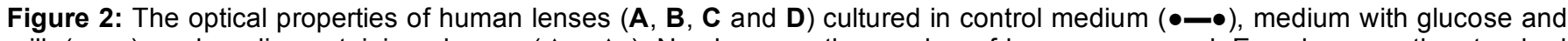
milk (o-0), and media containing glucose $(\triangle--\triangle)$. Numbers are the number of lenses measured. Error bars are the standard error of the mean. Opacity grades from pictures of (D) human and $(E)$ rat lenses as shown in Figure 3. 
A)

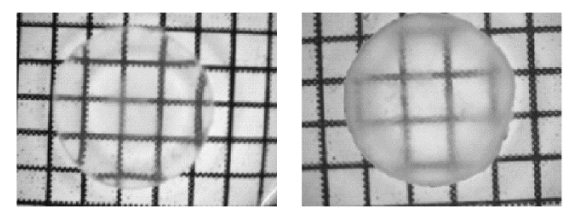

Grade 1: Clear
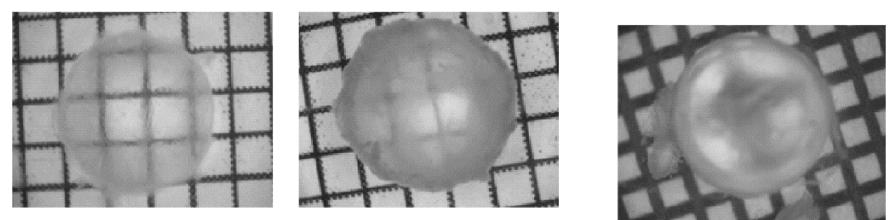

Grade 2: Slight Haze
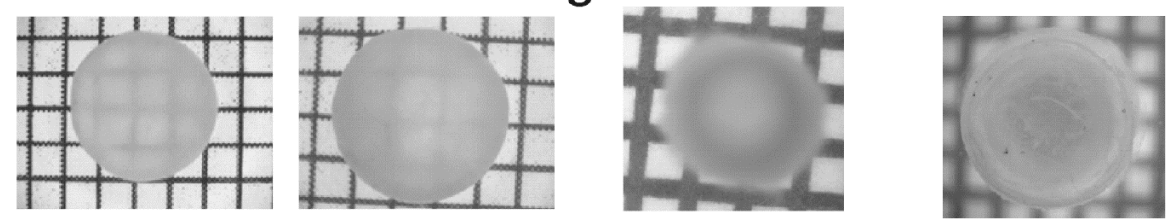

Grade 3: Lines Just Visible

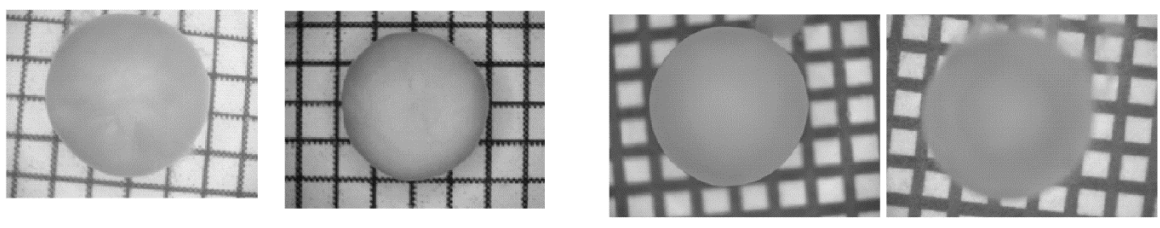

Grade 4: Completely Opaque

Figure 3: Grading guide used to grade $\mathbf{A}$ ) human lenses and $\mathbf{B}$ ) rat lens opacity in Figure $\mathbf{2 D}$ and $\mathbf{E}$.

A)

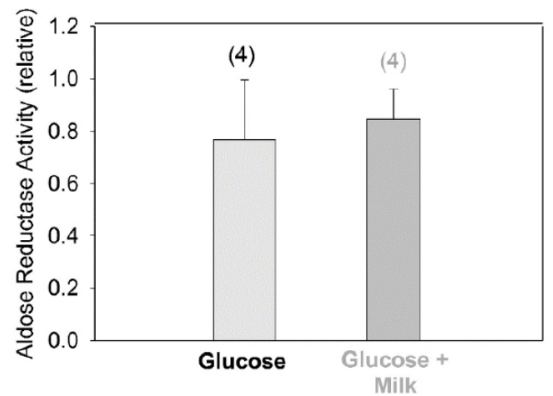

C)

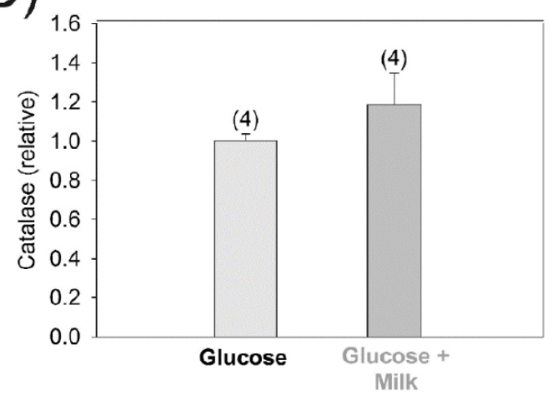

B)

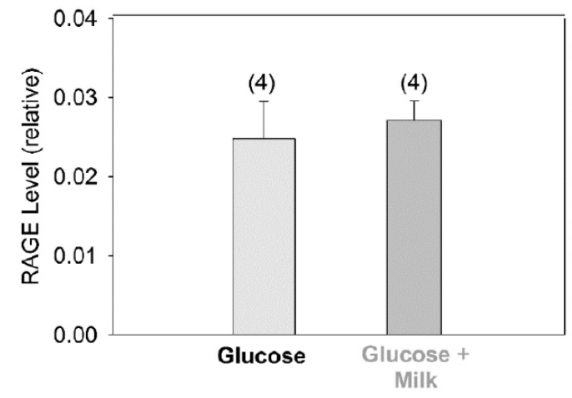

D)

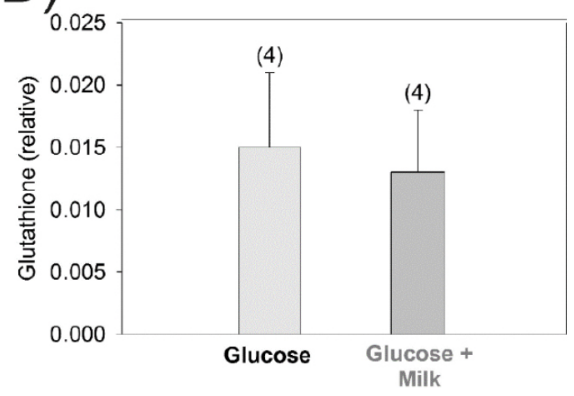

Figure 4: Assays on treated human lenses at four days in organ culture. Error bars are the standard error of the mean. Numbers are the number of lenses measured. 
A)

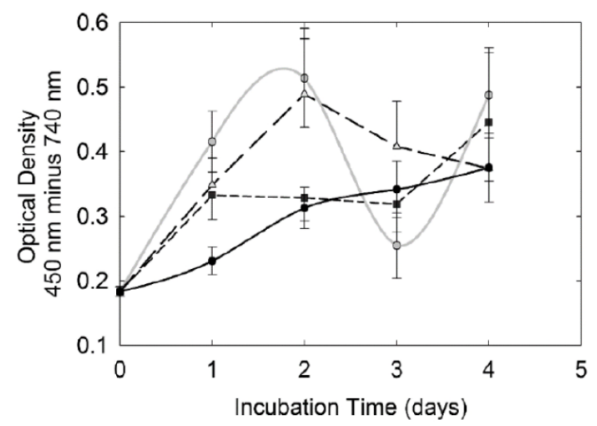

C)

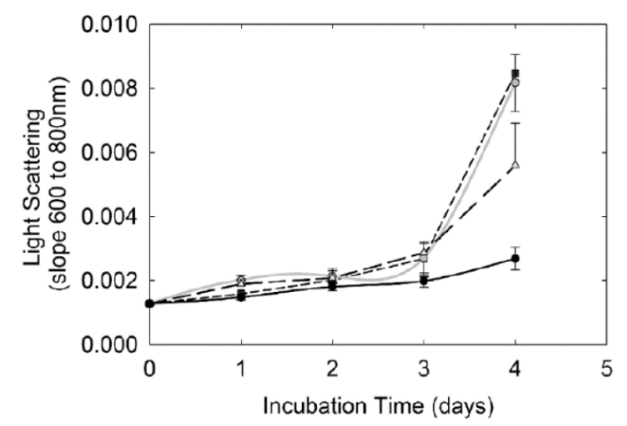

B)

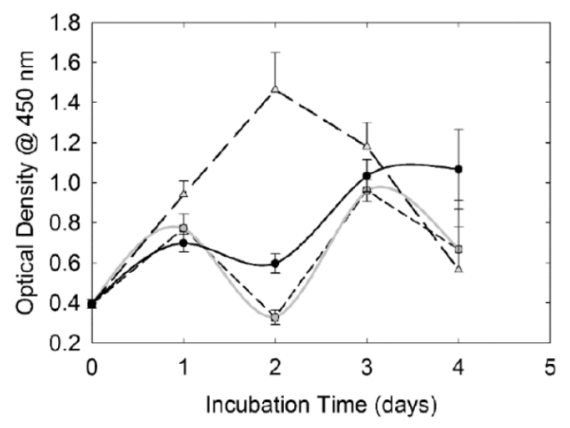

Figure 5: The optical properties of rat lenses organ cultured in control media $(\bullet-\bullet)$, media with glucose and milk (0-0), media containing glucose ( --- ) and media containing milk $(\triangle---\triangle)$. Error bars are the standard error of the mean. The number of lenses measured for lenses cultured for $0,1,2,3$ and 4 days were respectively: untreated lenses $71,18,19,19,13$; glucose treated $71,9,9,9,4$; glucose plus milk treated $71,8,8,8,4$; milk treated $71,9,9,9,3$.

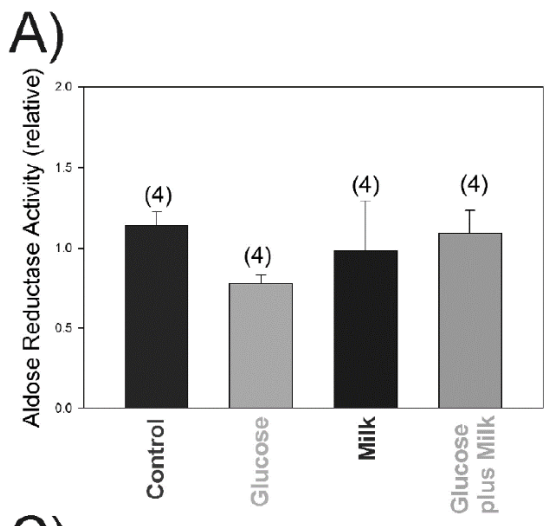

C)

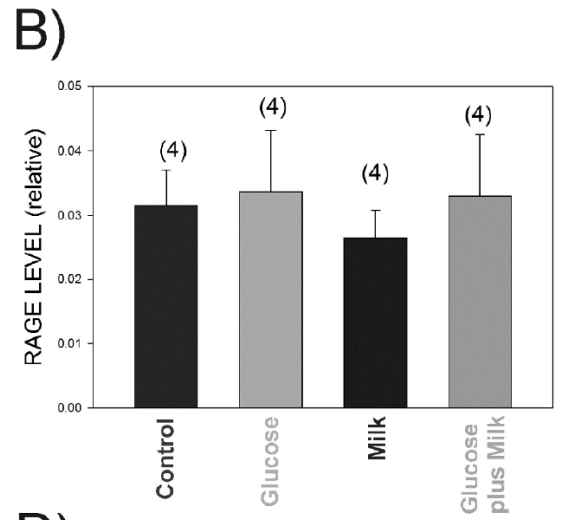

D)
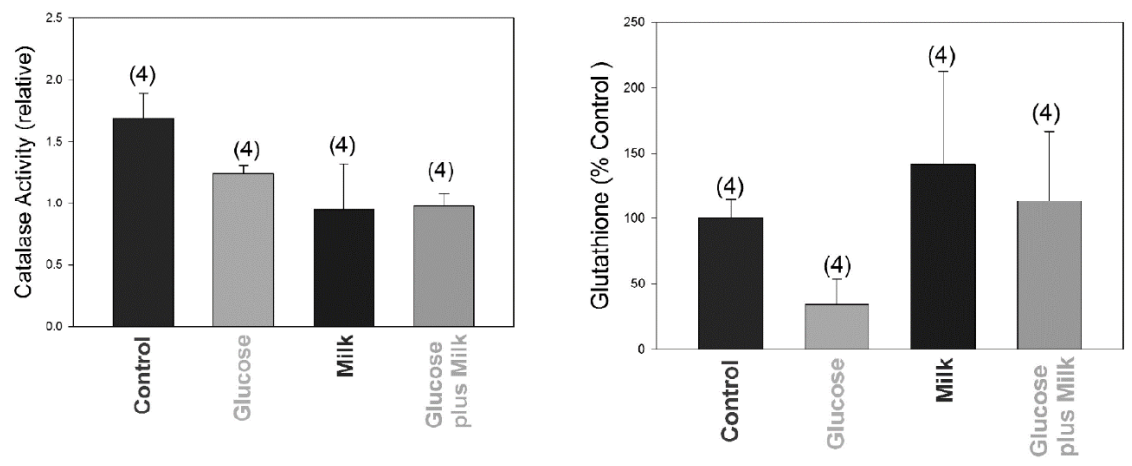

Figure 6: Assays on treated human lenses at four days in organ culture. Error bars are the standard error of the mean. Numbers are the number of lenses measured. Two rat lenses from the same group were pooled. 
compared with $R L c$ and rat lenses incubated with both glucose and camel milk (RLgm) (Figure 1B). As with human lenses (Figure 1A), a broad absorbance peak near $600 \mathrm{~nm}$ appeared in the spectra of RLg that was not present in the spectra of RLc but was not absent in RLgm (Figure 1B). At day two in organ culture, the O.D. at $450 \mathrm{~nm}$ (Figure 5B) and $\triangle$ O.D. (Figure 5A) were lower for RLc and rat lenses cultured with camel milk (RLm) when compared with RLg and RLgm. There was no significant difference $(P>0.05)$ between the O.D. and $\triangle$ O.D. for all rat lens cohorts at incubation times other than at two days. Except at an incubation time of four days, there was no difference in the light scattering between any of the cohorts (Figure 5C). However, on day four, the O.D. of RLC was significantly $(P<0.05)$ less than the other cohorts.

After four days in organ culture, aldose reductase activity (Figure 6A), catalase activity (Figure 6C) and glutathione levels (Figure 6D) for RLg, were attenuated compared with RLc. The level of RAGE was relatively unchanged for all rat lens cohorts (Figure 6B).

Rat lenses were graded by a blinded grader into four categories based on the opacity of the lens (Figure 3B). RLg were almost completely opaque after two days in organ culture (Figure 2E) and slightly more opaque than RLc. RLgm and RLm were about the same opacity grade as RLg (Figure $\mathbf{2 E}$ ).

\section{DISCUSSION}

The major finding of the current study was that camel milk present in the organ culture medium inhibited glucose-induced opacity, especially in human lenses. Because the etiology of diabetic cataract is multifactorial, complex, and not completely established, it is difficult to speculate on the mechanism whereby camel milk inhibits glucose cataract. Hyperglycemia is undoubtedly the initiating factor for diabetic cataract [26]. It is likely to involve osmotic stress and/or oxidation related metabolic changes $[26,27]$. Based on the author's studies, elevated levels of calcium are also likely to contribute to glucose cataract [28]. It is reasonable to speculate that osmotic stress causes mitochondria to produce reactive oxygen species which, in turn, oxidize membrane lipids $[22,23]$, increasing membrane permeability [29-32] causing an increase in intracellular calcium [28], which may activate lipases and proteases [33,34]. All of these factors contribute to changes in the gross and molecular structure of lenses resulting in increased light scattering, the major factor for the attenuation of light in glucose treated lenses [18]. Although not a major factor in the attenuation of light through the lens, camel milk in the medium of organ cultured human lenses decreased the absorbance bands between 550 and $700 \mathrm{~nm}$ that were induced by glucose. The finding from the current study that camel milk restored RLgm glutathione levels to that of RLc, suggests that the high ascorbate content of camel milk [3-6] may protect the lens from oxidative insult. The factors responsible for these observations are speculative. One possibility is that camel milk proteins coat the capsule protecting the lenses from the damaging effects of glucose. Future studies to determine the moieties in camel milk that are responsible for cataract inhibition are planned. The positive results from this initial in vitro study suggests that costlier and time consuming in vivo studies to determine the efficacy of camel milk in vivo are warranted.

The utility of using human lenses to test the efficacy of drugs and agents is promising. Because rat lenses become opaque relatively quickly (one day) they are less promising tools for organ culture experiments. Rat lenses differ significantly from human lenses so the use of human lens models is significantly advantageous over animal models $[19,20]$. Furthermore, it has been suggested that rat lenses are more susceptible to oxidation than human lenses due to their higher unsaturated glycolipid content [21-25].

In conclusion, camel milk present in the organ culture medium suppressed glucose-induced opacity, especially in human lenses, and restored the rat lens glutathione level to that of untreated rat lenses while causing a decrease in catalase activity. Because opacity is the ultimate end point for cataractous lens studies, quantitative assessment of absorbance and light scattering in lenses is essential for model studies.

\section{ACKNOWLEDGEMENTS}

Major support for this study was generously provided by the grant: Deanship for Scientific Research, Al Baha University, Saudi Arabia through the project No. 107-1436 (AHSA). Very minor support was obtained from other grants: an unrestricted grant from Research to Prevent Blindness, Inc. New York, NY, USA (DB), Aliza Williams received a fellowship from an Institutional Development Award (IDeA) from the National Institute of General Medical Sciences of the National Institutes of Health under grant number P20GM103436. Kayla Massay received a fellowship from the Physiology and Cardiovascular Minority 
Program and Collin Henry received a Medical School Student fellowship from the 'Summer Research Scholar Program', the University of Louisville, Louisville, KY, USA. There were no financial conflicts of interest for any of the authors.

\section{REFERENCES}

[1] Bourne RR, Stevens GA, White RA, et al. Causes of vision loss worldwide, 1990-2010: a systematic analysis. Lancet Glob Health 2013; 1: e339-349. https://doi.org/10.1016/S2214-109X(13)70113-X

[2] Li L, Wan $\mathrm{XH}$, Zhao $\mathrm{GH}$. Meta-analysis of the risk of cataract in type 2 diabetes. BMC Ophthalmol 2014; 14: 94. https://doi.org/10.1186/1471-2415-14-94

[3] Mohamed HE, Mousa HM, Beynen AC. Ascorbic acid concentrations in milk from Sudanese camels. J Animal Physiol Anim Nutr 2005; 89: 35-37. https://doi.org/10.1111/j.1439-0396.2004.00507.x

[4] Mehaia MA, Al-Kahnal MA. Studies on camel milk goat milk proteins: nitrogen distribution and amino acid composition. Nutr Rep Int 1989; 39: 351-357.

[5] Farah Z, Rettenmaier R, Atkins D. Vitamin content of camel milk. Int J Vitam Nutr Res 1992; 62: 30-33.

[6] Knosess $\mathrm{KH}$. The camel as meat and milk animal. World Anim Rev 1977; 22: 39-44.

[7] Weikel KA, Garber C, Baburins A, Taylor A. Nutritional modulation of cataract. Nutr Rev 2014; 72: 30-47. https://doi.org/10.1111/nure.12077

[8] Zibaee S, Hosseini SMA, Yousefi M, Taghipour A, Kiani MA, Noras MR. Nutritional and Therapeutic Characteristics of Camel Milk in Children: A Systematic Review. Electron Physician 2015; 7: 1523-1528. https://doi.org/10.19082/1523

[9] Agrawal R, Dogra R, Mohta N, Tiwari R, Singhal S, Sultania S. Beneficial effect of camel milk in diabetic nephropathy. Acta Biomed 2010; 80: 131-134.

[10] Ehlayel MS, Hazeima KA, Al-Mesaifri F, Bener A. Camel milk: an alternative for cow's milk allergy in children. Allergy Asthma Proc 2011; 32: 255-258. https://doi.org/10.2500/aap.2011.32.3429

[11] Conesa C, Sanchez L, Rota C, et al., Isolation of lactoferrin from milk of different species: Calorimetric and antimicrobial studies. Comp Bioch Physiol Biochem Mol Biol 2008; 150: 131-139.

https://doi.org/10.1016/j.cbpb.2008.02.005

[12] Saadaoui B, Henry C, Khorchani T, Mars M, Martin P, Cebo C. Proteomics of the milk fat globule membrane from Camelus dromedarius. Proteomics 2013; 13: 1180-1184. https://doi.org/10.1002/pmic.201200113

[13] Yang $\mathrm{Y}$, Zheng N, Wang W, et al. N-glycosylation proteomic characterization and cross-species comparison of milk fat globule membrane proteins from mammals. Proteomics 2016; 16: 2792-2800. https://doi.org/10.1002/pmic.201500361

[14] Casado B, Affolter M, Kussmann M. OMICS-rooted studies of milk proteins, oligosaccharides and lipids. J Proteomics 2009; 73: 196-208.

https://doi.org/10.1016/j.jprot.2009.09.018

[15] Spertino S, Cipriani V, De Angelis C, et al. Proteome profile and biological activity of caprine, bovine and human milk fat globules. Mol Biosyst 2012; 8: 967-974 https://doi.org/10.1039/C2MB05400K

[16] Peterson JA, Scallan CD, Ceriani RL, Hamosh M. Structural and functional aspects of three major glycoproteins of the human milk fat globule membrane. Adv Exp Med Biol 2001; 501: 179-187.

https://doi.org/10.1007/978-1-4615-1371-1 23

[17] Alghamd AHS, Mohamed $H$, Sledge S, Borchman D. Absorbance and Light Scattering of Lenses Organ Cultured With Glucose. Curr Eye Res In 2018; 6: 1-6. https://doi.org/10.1080/02713683.2018.1485953

[18] Bree M, Borchman D. Optical Properties of Rat, Porcine and Human Lenses and Organ Culture. Exp Eye Res 2018; 170: 67-75.

https://doi.org/10.1016/j.exer.2018.02.016

[19] Truscott RJW. Age-related nuclear cataract-oxidation is the key. Exp Eye Res 2005; 80: 709-725. https://doi.org/10.1016/j.exer.2004.12.007

[20] Borchman D, Yappert MC, Afzal M. Lens lipids and maximum lifespan. Exp Eye Res 2004; 79: 761-768. https://doi.org/10.1016/j.exer.2004.04.004

[21] Huang L, Yappert MC, Miller JJ, Borchman D. Thyroxine Ameliorates Oxidative Stress by Inducing Lipid Compositional Changes in Human Lens Epithelial Cells. Invest Ophthalmol Vis Sci 2007; 48: 3698-3704. https://doi.org/10.1167/iovs.06-0908

[22] Huang L, Yappert MC, Jumblatt M, Borchman D. Hyperoxia and Thyroxine-Treatment and the Relationships Between Reactive Oxygen Species Generation, Mitochodrial Membrane Potential and Cardiolipin in Human Lens Epithelial Cell Cultures. Curr Eye Res 2008; 33: 575-586. https://doi.org/10.1080/02713680802167554

[23] Huang L, Tang D, Yappert MC, Borchman D. Oxidation Induced Changes in Human Lens Epithelial Cells. 2. Mitochondria and the Generation of Reactive Oxygen Species. Free Rad Biol Med 2006; 41: 926-936. https://doi.org/10.1016/j.freeradbiomed.2006.05.023

[24] Huang L, Estrada R, Yappert MC, Borchman D. Oxidation Induced Changes in Human Lens Epithelial Cells. 1. Phospholipids. Free Rad Biol Med 2006; 41: 1425-1432. https://doi.org/10.1016/j.freeradbiomed.2006.07.022

[25] Borchman D, Stimmelmayr R, George JC. Whales, Lifespan, Phospholipids and Cataracts. J Lipid Res 2017; 58: 22892298.

https://doi.org/10.1194/jlr.M079368

[26] Pollreisz A, Schmidt-Erfurth U. Diabetic cataractpathogenesis, epidemiology and treatment. J Ophthalmol 2010; 2010: 608751. https://doi.org/10.1155/2010/608751

[27] Lu Q, Yang T, Zhang M, Du L, Liu L, Zhang N, Guo H, Zhang F, Hu G, Yin X. Preventative effects of ginkgo biloba extract (EGb761) on high glucose-cultured opacity of rat lens. Phytother Res 2014; 28: 767-773. https://doi.org/10.1002/ptr.5060

[28] Tang D, Borchman D, Yappert MC, Vrenson GFJM. Influence of age, diabetes, and cataract on lipid-calcium and proteincalcium relationships in human lenses. Invest Ophthalmol Vis Sci 2003; 44: 2059-2066. https://doi.org/10.1167/iovs.02-0345

[29] Pasino M, Maraini G. Cation pump activity and membrane permeability in human senile cataractous lenses. Exp Eye Res. 1982; 34: 887-893. https://doi.org/10.1016/0014-4835(82)90068-9

[30] Lucas VA, Duncan G, Davies PD. Membrane permeability characteristics of perfused human senile cataractous lenses. Exp Eye Res 1986; 42: 151-165. https://doi.org/10.1016/0014-4835(86)90039-4

[31] Duncan G, Jacob TJ. Human cataract formation. CIBA Found Symp 1984; 106: 132-148.

[32] Gandolfi SA, Tomba CM, Maraini G. 86-Rb Efflux in normal and cataractous human lenses. Curr Eye Res 1985; 4: 753758.

https://doi.org/10.3109/02713688509020030 
[33] Yoshida H, Murachi T, Tsukahara J. Limited proteolysis of bovine lens alpha crystallin by calpain, a $\mathrm{Ca} 2$ dependent cysteine proteinase, isolated from the same tissue. Biochim Biophys Acta 1984; 798: 252-259.

https://doi.org/10.1016/0304-4165(84)90313-1
[34] Ireland $\mathrm{M}$, Maisel $\mathrm{H}$. Evidence for a $\mathrm{Ca}$ activated protease specific for lens intermediate filaments. Curr Eye Res 1984; 3: 423-429.

https://doi.org/10.3109/02713688408997229

Received on 08-06-2018

Accepted on 29-06-2018

Published on 16-08-2018

DOI: https://doi.org/10.6000/1929-5634.2018.07.02.1

(C) 2018 Alghamdi et al.; Licensee Lifescience Global.

This is an open access article licensed under the terms of the Creative Commons Attribution Non-Commercial License (http://creativecommons.org/licenses/by-nc/3.0/) which permits unrestricted, non-commercial use, distribution and reproduction in any medium, provided the work is properly cited. 